\title{
Reversed Genetic Algorithms for Generation of Bijective S-boxes with Good Cryptographic Properties
}

\author{
Georgi Ivanov $^{1}$, Nikolay Nikolov ${ }^{2}$, and Svetla Nikova ${ }^{3}$ \\ 1 Institute of Mathematics and Informatics, Bulgarian Academy of Sciences \\ 2 Institute of Mathematics and Informatics, Bulgarian Academy of Sciences \\ 3 KU Leuven, Dept. ESAT/COSIC and iMinds, Belgium
}

\begin{abstract}
Often S-boxes are the only nonlinear component in a block cipher and as such play an important role in ensuring its resistance to cryptanalysis. Cryptographic properties and constructions of S-boxes have been studied for many years. The most common techniques for constructing S-boxes are: algebraic constructions, pseudo-random generation and a variety of heuristic approaches. Among the latter are the genetic algorithms. In this paper, a genetic algorithm working in a reversed way is proposed. Using the algorithm we can rapidly and repeatedly generate a large number of strong bijective S-boxes of each dimension from $(8 \times 8)$ to $(16 \times 16)$, which have sub-optimal properties close to the ones of S-boxes based on finite field inversion, but have more complex algebraic structure and possess no linear redundancy.
\end{abstract}

Keywords: Genetic Algorithms, S-boxes, Nonlinearity

\section{Introduction}

Most of the modern block ciphers have one or more non-linear components providing the effect of confusion [31], which is of vital importance for the strength of the cipher. The most often used non-linear components are $n$ to $m$ Boolean mappings, called S-boxes. Among them, the bijective S-boxes are particularly interesting. Block ciphers have to be resistant to linear and differential cryptanalysis $[3,4,17]$. It is well known that the weight of a differential trail is equal to or larger than the product of the number of active S-boxes and the minimum (differential) weight per S-box (analogous for the linear trails). There are two known approaches for eliminating the low-weight trails - either by increasing the number of active S-boxes or by choosing stronger S-boxes. The number of active S-boxes can be increased by designing stronger linear layer, e.g., by using the wide trail strategy [10]. Larger S-boxes can be stronger since the minimum differential weight of an S-box is limited by its size. In this paper we focus only on the second approach. In order to be suitable for use in cryptographic applications S-boxes should possess high nonlinearity and low differential uniformity. In addition, cryptographically strong S-boxes should also satisfy cryptographic criteria such as: regularity (balancedness) and high algebraic degree, as well as they should possess as few fixed points and linear redundancy as possible.

\subsection{Motivation}

Large S-boxes can fast improve the security (in the black box model) of a block cipher. In addition, the results in [16] indicate that the resistance to side-channel analysis could also be improved by increasing the size of the S-box. However, finding or constructing such big and cryptographically strong S-boxes is still an open problem. 
The approaches for S-box generation available in the literature could be divided into three main streams: algebraic constructions, pseudo-random generation and heuristic techniques. The first approach is based on S-box generation according to mathematical principles. For example the S-box of AES [10] is constructed with inverse mapping followed by an affine transformation in the finite field. S-boxes based on inversion in the finite field $G F\left(2^{n}\right)$ are known to achieve the best values found for the nonlinearity and differential uniformity $\left(N_{i n v}=2^{n-1}-2^{\frac{n}{2}}\right.$ and $\delta_{i n v}=4$ respectively, [24]). Although these values are not the optimal values theoretically possible $\left(N_{o p t}=2^{n-1}-2^{\frac{n}{2}-1}\right.$ and $\left.\delta_{o p t}=2,[23]\right)$ and filling up the gap between them and their known theoretical bounds is an open problem in cryptography, it is believed that the finite field inversion-based S-boxes are the optimal ones that exist with respect to the simultaneous satisfaction of all targeted cryptographic criteria. A new method for constructing 4-uniform permutations has recently been proposed in [26,27].

The second approach is based on using some pseudo-random generation to generate Sboxes. However, to find good S-boxes quickly becomes infeasible as the size of the input space increases. Also, the probability of finding really strong S-boxes is very small. For example, in the case of $(8 \times 8)$ S-boxes, the highest value for nonlinearity found is $98-100$ [20,21], which is rather low compared to the value of 112 for the finite field inversion-based case.

The third approach uses heuristic algorithms in a process of iteratively improving given Sbox or S-boxes with respect to one or more properties. Because these algorithms use directed search methods, unlike the algebraic constructions they are able to produce a large number of S-boxes, which are not optimal but sub-optimal. Specific heuristic techniques include the hill climbing method, the simulated annealing method, the genetic algorithm or a combination of these. For example the highest nonlinearity achieved by: the hill climbing method is 100 [20], the simulated annealing method is 102 [8], and by a special genetic algorithm is 104 [32]. Recently in [15], values of 104 have been achieved for the nonlinearity by a method, referred as the modified gradient descent, which is based on swapping a number of values in a permutation.

The construction of a large number of good bijective S-boxes quickly becomes a hard job as their size increases. As the number of input variables $n$ increases by one, the number of Boolean functions in the space increases by a factor of $2^{2^{n}}$. That is why, except for the algebraic construction method, the other two methods fail short already when $n=8$. Therefore, it is a challenge to improve the results for size 8 , and even more, to go for larger S-boxes.

\subsection{Contribution}

Often it is desired (e.g. for efficient implementations) to find a large set of strong bijective S-boxes possessing the same cryptographic properties and to allow the designer to freely choose the S-box within this set to be used in a cryptographic algorithm. This cannot be achieved using algebraic constructions and pseudo-random generation methods, since either it will not be possible to find many S-boxes or they will not be strong enough. What concerns the known heuristic techniques, the conventional approach from bottom to the top, where usually one starts with some initial set of random S-boxes and tries iteratively to improve them, is not quite suitable. The obtained in this way set of values corresponding to the set of targeting criteria is not close enough to the set of values of finite field inversion-based S-boxes. Furthermore, when one searches for a set of good S-boxes which in addition have to be big - from $(8 \times 8)$ to $(16 \times 16)$, all generation methods known are quite impractical, due to the huge time and memory resources needed for the computations. Therefore, in this paper we propose a new method based on a genetic algorithm which is working in a "reversed" way. 
More precisely, the algorithm starts from an initial pool of S-boxes based on inversion in the finite field and searches for S-boxes which are close to them with respect to the targeted set of cryptographic criteria. The detailed description is provided in Section 4. Applying the new algorithm we repeatedly and rapidly obtain a large set of S-boxes which are:

1. of dimensions from $(8 \times 8)$ to $(16 \times 16)$;

2. have properties close to the best properties found of S-boxes based on finite field inversion;

3. have more complex algebraic structure;

4. possess little or no linear redundancy.

For the case of $(8 \times 8)$ bijective S-boxes the algorithm outputs thousands of S-boxes with nonlinearities 106, 108, 110 and $112\left(=N_{\text {inv }}\right)$ after 2 days work on a cluster with 32 cores. S-boxes with nonlinearity 110 and possessing zero (i.e. no linear) redundancy as well as Sboxes with nonlinearity 112 which have incomplete redundancy are not reported till now in the literature.

For the case of $(16 \times 16)$ bijective S-boxes 50 S-boxes with values of nonlinearity $32400=$ $N_{i n v}-112$ are obtained $\left(N_{i n v}=2^{n-1}-2^{\frac{n}{2}}=32512\right)$ after 3 months work on the same computer. With nonlinearities closer to $N_{i n v}$, for example $N_{S}=N_{i n v}-36=32476$, 50 Sboxes are obtained for less than a month. In fact, thanks to the "reversed" way the algorithm works, it can output S-boxes with each possible value of nonlinearity up to $N_{i n v}$. Furthermore, the closer to $N_{i n v}$ the values of the nonlinearity are, the faster the algorithm works. We are not aware of any constructions of S-boxes with size bigger than 8, obtained with either pseudorandom generation or heuristic techniques. Thus, it is the first time when such large S-boxes with good cryptographic properties are generated. In Table 1 we compare our results with the state of the art results for the case $n=8$.

Table 1: A comparison between the cryptographic properties of $(8 \times 8)$ bijective S-boxes obtained by various generation methods (NR stands for "not reported")

\begin{tabular}{|l|c|c|c|c|c|c|}
\hline Methods/Properties & $N_{S}$ & $\operatorname{deg}(S)$ & $A C(S)_{\max }$ & $\delta$-uniformity & Fixed points & Linear Redundancy \\
\hline Pseudo-random S-BOX [20], [21] & 98 & $\mathrm{NR}$ & $\mathrm{NR}$ & $\mathrm{NR}$ & $\mathrm{NR}$ & NR (usually zero) \\
\hline Finite Field Inversion [24] & 112 & 7 & 32 & 4 & 2 & complete \\
\hline Hill Climbing [20] & 100 & $\mathrm{NR}$ & $\mathrm{NR}$ & $\mathrm{NR}$ & $\mathrm{NR}$ & NR \\
\hline GA/HC [21] & 100 & $\mathrm{NR}$ & $\mathrm{NR}$ & $\mathrm{NR}$ & $\mathrm{NR}$ & NR \\
\hline Simulated Annealing [8] & 102 & $\mathrm{NR}$ & 80 & $\mathrm{NR}$ & $\mathrm{NR}$ & NR \\
\hline GaT [32] & 104 & $\mathrm{NR}$ & $\mathrm{NR}$ & $\mathrm{NR}$ & $\mathrm{NR}$ & NR \\
\hline Tweaking [12] & 106 & 7 & 56 & 6 & $\mathrm{NR}$ & zero \\
\hline Gradient descent method [15] & 104 & 7 & 80 & 8 & 0 & NR \\
\hline 4-uniform permutations method [26,27] & 98 & $\mathrm{NR}$ & $\mathrm{NR}$ & 4 & $\mathrm{NR}$ & NR \\
\hline GA1 [this paper] & 106 & 6 & 56 & 6 & 2 & zero \\
GA1 [this paper] & 108 & 6 & 48 & 6 & 0 & zero \\
\hline GA2 [this paper] & 110 & 7 & 40 & 6 & 0 & zero \\
GA2 [this paper] & 112 & 7 & 32 & 6 & 0 & some (147 of 255) \\
\hline
\end{tabular}

Note that algebraic immunity is also included in the target set of criteria for the Gradient descent method. The reported value for algebraic immunity is 3 [15]. We do not include algebraic immunity in our target set of criteria. 


\section{Preliminaries}

In this section we will briefly recall some of the basic definitions and properties of Boolean functions. For a comprehensive survey on Boolean functions we refer to $[5,6]$.

Let the substitution table (S-box) of an n-binary input into m-binary output mapping is denoted by $S$. Then $S: \mathbb{B}^{n} \rightarrow \mathbb{B}^{m}$ and to each $x=\left(x_{1}, x_{2}, \ldots, x_{n}\right) \in \mathbb{B}^{n}$ some $y=$ $\left(y_{1}, y_{2}, \ldots, y_{m}\right) \in \mathbb{B}^{m}$ is assigned by $S(x)=y$, where $\mathbb{B}=\{0,1\}$ is the 1-dimensional Boolean space. Clearly, $S$ can be considered as a vectorial Boolean function consisting of $m$ individual Boolean functions $f_{1}, f_{2}, \ldots, f_{m}$, where $f_{i}: \mathbb{B}^{n} \rightarrow \mathbb{B}$ and $f_{i}(x)=y_{i}$ for $i=1,2, \ldots, m$. These functions are called coordinate Boolean functions of the S-box $S$ and it is well known that most of the desirable cryptographic properties of $S$ can be defined in terms of their linear combinations. S-box coordinate Boolean functions and all their linear combinations are referred as the S-box component Boolean functions.

\subsection{Boolean functions}

A Boolean function can be represented by a truth table, which is the binary output vector of the function containing $2^{n}$ elements. We obtain the polarity truth table when instead of $f(x)$, the signed function $\widehat{f}(x)=(-1)^{f(x)}$ is considered. Another way of representing a Boolean function is by means of its algebraic normal form $(A N F)$ :

$$
f(x)=a_{0} \oplus a_{1} x_{1} \oplus a_{2} x_{2} \oplus \ldots \oplus a_{n} x_{n} \oplus a_{1,2} x_{1} x_{2} \oplus \ldots \oplus a_{1,2, \ldots, n} x_{1} x_{2} \ldots x_{n},
$$

where the coefficients $a_{I} \in \mathbb{B}, I \subseteq\{1,2, \ldots, n\}$. The algebraic degree of an $n$-variable Boolean function $f(x)$, denoted by $\operatorname{deg}(f)$, is the number of variables of the largest product term of the function's $A N F$ having a non-zero coefficient.

Two $n$-variable Boolean functions $f(x)$ and $g(x)$ belong to the same equivalence class (or are affine equivalent) if and only if there exist some invertible $(n \times n)$ binary matrix $A$, vectors $b, c \in \mathbb{B}^{n}$ and a scalar $d \in \mathbb{B}$, such that $g(x)=f(A x \oplus b) \oplus\langle c, x>\oplus d$.

The Walsh-Hadamard transform (WHT) of an $n$-variable Boolean function $\widehat{f}(x)$, denoted by $\widehat{F}_{f}(w)$, is defined by:

$$
\widehat{F}_{f}(w)=\sum_{x \in \mathbb{B}^{n}} \widehat{f}(x)(-1)^{<w, x>}=\sum_{x \in \mathbb{B}^{n}}(-1)^{f(x) \oplus<w, x>}=\sum_{x \in \mathbb{B}^{n}} \widehat{f}(x) \widehat{l}_{w}(x),
$$

where $\widehat{l}_{w}(x)$ is the signed function of the linear function $l_{w}(x)=<w, x>$.

Thus, $\forall w \in \mathbb{B}^{n}, \widehat{F}_{f}(w) \in\left[-2^{n}, 2^{n}\right] . \widehat{F}_{f}(w)$ is called a spectral Walsh coefficient and the real-valued vector of all $2^{n}$ Walsh coefficients is referred to as the WHT Spectrum. We denote the maximum absolute value taken by the $W H T$ by $W H T_{\max }(f)=\max _{\left(w \in \mathbb{B}^{n}\right)}\left|\widehat{F}_{f}(w)\right|$.

Some of the most important cryptographic properties that a Boolean function should possess are defined below. An $n$-variable Boolean function $f(x)$ is called balanced if $w_{H}(f)=$ $2^{n-1}$, where by $w_{H}(f)$ is denoted the Hamming weight of $f$. The nonlinearity of an $n$-variable Boolean function $f(x)$, denoted by $N_{f}$, is the minimum distance to the set of all $n$-variable affine Boolean functions $A(n)$. It is given by $N_{f}=\left[2^{n}-W H T_{\max }(f)\right] / 2$. The autocorrelation transform $(A C T)$ of $\widehat{f}(x)$, denoted by $\widehat{r}_{f}(\alpha)$, taken with respect to a vector $\alpha \in \mathbb{B}^{n}$ is defined by:

$$
\widehat{r}_{f}(\alpha)=\sum_{x \in \mathbb{B}^{n}}(-1)^{f(x) \oplus f(x \oplus \alpha)}=\sum_{x \in \mathbb{B}^{n}} \widehat{f}(x) \widehat{f}(x \oplus \alpha)
$$


Thus, $\forall \alpha \in \mathbb{B}^{n}, \widehat{r}_{f}(\alpha) \in\left[-2^{n}, 2^{n}\right]$ and $\widehat{r}_{f}(0)=2^{n}$. The $\widehat{r}_{f}(\alpha)$ is called spectral autocorrelation coefficient and the real-valued vector of all $2^{n}$ autocorrelation coefficients representing the $A C T$ of the function is referred to as its ACT Spectrum.

The maximum $A C T$ value or absolute indicator of an $n$-variable Boolean function in polarity form $\widehat{f}(x)$, denoted by $A C_{\max }(f)$, is defined by: $A C_{\max }=\max _{\left(\alpha \in \mathbb{B}^{n} \backslash\{0\}\right)}\left|\widehat{r}_{f}(\alpha)\right|$.

The results of Meier and Staffelbach [19] and of Preneel [25] show that some of the most important cryptographic characteristics of Boolean functions as algebraic degree, nonlinearity and absolute indicator are invariant under affine transformations, while in other, like WHT and $A C T$, the effect produced by applying affine transformations is permutation in the values of the spectral coefficients, and some change in their signs. Anyway, their magnitudes are not affected by affine transformations. In other words, for any two affine equivalent $n$-variable Boolean functions $f$ and $g$, is true that $\operatorname{deg}(g)=\operatorname{deg}(f), W H T_{\max }(g)=W H T_{\max }(f), N_{g}=$ $N_{f}$ and $A C_{\max }(g)=A C_{\max }(f)$.

\subsection{Vectorial Boolean functions}

The properties of Boolean functions discussed in the previous section can be extended to the case of vectorial Boolean functions (S-boxes). There are some conceptual similarities in the transition from the single-output to the multi-output case but there are also some essential differences in the manner by which the S-boxes properties are derived. It is important to note that it is not sufficient only to consider the coordinate Boolean function properties when considering S-box cryptographic properties but also their linear combinations.

To avoid trivial statistical attacks, a good S-box should be a regular (balanced) mapping. An $(n \times m)$ S-box $S$ with $n \geq m$ is said to be regular if for each its output $y \in \mathbb{B}^{m}$ there are exactly $2^{n-m}$ inputs that are mapped to $y$. If the S-box is not regular, some outputs appear more often than others when the input to the S-box is randomly chosen and the bias can be exploited by the cryptanalyst. An $(n \times m)$ S-box with $n \geq m$ is regular if and only if all non-zero its component Boolean functions are balanced [30].

Linear cryptanalysis [17], is a known-plaintext attack which is trying to approximate the relationship between plaintext, ciphertext and the key bits by constructing a linear expression and then evaluating the probability $P$ attached to this expression. Among all possible expressions, those which achieve highest/lowest probabilities to be the correct are the best linear/affine approximations. Thus, if all the probabilities $P$ are approximately equal to $\frac{1}{2}$, the cipher will be resistant to linear and affine approximation.

An $(n \times m)$ S-box improves the immunity against linear cryptanalysis if in its linear approximation table (LAT) all the entries magnitudes are as small as possible. In [29] it is shown that the latter is equivalent to the statement that the nonlinearity of each non-zero S-box component Boolean function should be as high as possible. The nonlinearity of an $(n \times m)$ S-box $S$, denoted by $N_{S}$, is defined as the minimum nonlinearity of each of the component Boolean functions excluding the zero one. It can be expressed as:

$N_{S}=\min _{\left(c=\left(c_{1}, c_{2}, \ldots, c_{m}\right) \in \mathbb{B}^{m} \backslash\{0\}\right)} N_{c_{1} f_{1} \oplus c_{2} f_{2} \oplus \ldots \oplus c_{m} f_{m}}$.

In order to resist low order approximation attacks each S-box must have an algebraic degree as high as possible $([14,22])$. The (minimal) algebraic degree of an $(n \times m)$ S-box $S$, denoted by $\operatorname{deg}(S)$, is defined as the minimum algebraic degree of each of its non-trivial component Boolean functions. It can be expressed as follows:

$$
\operatorname{deg}(S)=\min _{c=\left(c_{1}, c_{2}, \ldots, c_{m}\right) \in \mathbb{B}^{m} \backslash\{0\}} \operatorname{deg}\left(c_{1} f_{1} \oplus c_{2} f_{2} \oplus \ldots \oplus c_{m} f_{m}\right) .
$$


Differential cryptanalysis, introduced by Biham and Shamir [3] is applied to block ciphers as a chosen-plaintext attack, which consists in finding relationships between plaintext differences and their corresponding ciphertext differences in order to gain knowledge of the key bits. The differential uniformity of an $(n \times m)$ S-box $S$ with $n \geq m$, denoted by $\delta$, is defined as the largest value present in its difference distribution table (DDT) not counting the first entry in the first row. That is, $\delta=\max _{\alpha \in \mathbb{B}^{n} \backslash\{0\}} \max _{\beta \in \mathbb{B}^{m}}\left|\left\{x \in \mathbb{B}^{n} \mid S(x) \oplus S(x \oplus \alpha)=\beta\right\}\right|$. Then, $S$ is said to be differentially $\delta$-uniform.

Thus, a necessary condition for an S-box to improve the resistance against differential cryptanalysis is its DDT not to contain entries with large values ( $D D T$ to be flat) or equivalently the differential uniformity $\delta$ to be as small as possible $[1,7,11]$. In [34] the relation $A C(S)=D D T . H_{m}$ has been shown, where $H_{m}$ is the $\left(2^{m} \times 2^{m}\right)$ Sylvester-Hadamard matrix [18] and $A C(S)$ is the $\left(2^{n} \times 2^{m}\right)$ autocorrelation matrix of $S$ which columns represent the autocorrelation functions of all component Boolean functions of $S$. Also, in [34] a lower bound on the differential uniformity $\delta$ of $S$ is given, involving the maximum absolute value in $A C(S): \delta \geq 2^{n-m}+2^{-m} A C(S)_{\max }$, where by $A C(S)_{\max }$ it is denoted the maximum absolute indicator among the absolute indicators of all non-trivial component Boolean functions of $S$. That is, $A C(S)_{\max }=\max _{c=\left(c_{1}, c_{2}, \ldots, c_{m}\right) \in \mathbb{B}^{m} \backslash\{0\}}\left|\widehat{r}_{c_{1} f_{1} \oplus c_{2} f_{2} \oplus \ldots \oplus c_{1} f_{m}}(\alpha)\right|$.

Clearly $\delta \in\left[2^{n-m}, 2^{n}\right]$. If $A C(S)_{\max }$ is 0 , which is known to be achieved by the perfect nonlinear (bent) functions $[19,28]$, then $\delta$ will have the minimum value possible. Thus, small value of $\delta$ implies a small value for $A C(S)_{\max }$ and hence, minimizing the overall autocorrelation of S-boxes, in terms of their $A C(S)_{\max }$, will help in providing resistance to differential cryptanalysis. Considering that $\delta$ is always even, then in the case of bijective S-boxes $(n=m)$ can be concluded that the smallest possible value of $\delta$ is 2 . However, in $[3,4]$ it is pointed out that the condition of the $D D T$ of an S-box being flat is not sufficient for the S-box to resist differential cryptanalysis. In addition, the DDT should also contain as less non-zero entries as possible in its first column. In the case of bijective S-boxes this additional requirement is always fulfilled as the first column of the $D D T$ contains only zeros except for the first entry.

Taking into consideration the mentioned cryptographic properties of S-boxes and the properties of the coordinate Boolean functions and their linear combinations, we decided to target the following set of cryptographic criteria for a good bijective S-box $S$ :

1. Minimization of the largest non-trivial value in the $L A T$ of $S$ or $\Longleftrightarrow$

Maximization of the nonlinearity of $S, N_{S}$

2. Maximization of the algebraic degree of $S, \operatorname{deg}(S)$.

3. Minimization of the largest non-trivial value $\delta$ in the $D D T$ of $S$.

4. Minimization of the maximum absolute autocorrelation of $S, A C(S)_{\max }$.

5. Non-existence of fixed points.

6. Non-possession of linear redundancy.

We add Criterion 5 to the targeting set of cryptographic criteria as pointed out in [10], although we are not aware of any cryptanalytic attacks in the literature which takes advantage of the existence of fixed points in S-boxes.

In [12], Fuller and Millan proposed a new criterion to be added to the above set, the "non-possession of linear redundancy", which is defined bellow. We add it to the targeted set as the Criterion 6.

Definition 1. An $(n \times m) S$-box $S$ will possess linear redundancy if there are at least two Boolean functions $g(x)=a_{1} f_{1} \oplus a_{2} f_{2} \oplus \ldots \oplus a_{m} f_{m}$ and $h(x)=b_{1} f_{1} \oplus b_{2} f_{2} \oplus \ldots \oplus b_{m} f_{m}$ with $a=\left(a_{1}, a_{2}, \ldots, a_{m}\right)$ and $b=\left(b_{1}, b_{2}, \ldots, b_{m}\right) \in \mathbb{B}^{m} \backslash\{0\}$, which are affine equivalent. 
When all component Boolean functions of $S$ (not counting the zero linear combination) are affine equivalent, $S$ will possess complete linear redundancy. On the contrary, when no two affine equivalent non-zero linear combinations of the coordinate Boolean functions exists, $S$ will possess zero linear redundancy. Small S-boxes will always have linear redundancy because of the few available equivalence classes for smaller $n$. However, for larger S-boxes, as the number of equivalence classes becomes quickly infeasible, the presence of linear redundancy is claimed to be an indicator for non-randomness and thus a potential source of new cryptanalysis [12].

When optimizing Boolean functions for several cryptographic criteria simultaneously it is known that trade-offs exist. For example, the perfect nonlinear (bent) functions, which are known to achieve the highest possible value of nonlinearity $2^{n-1}-2^{\frac{n-2}{2}}$ and the lowest $\delta$-uniformity of 2, exist only for even $n$, but they are never balanced and always have small algebraic degree $\left(\leq \frac{n}{2}\right)$. Such trade-offs effect the combined properties of an S-box.

\section{Generation Methods}

The available in the literature techniques for S-box generation could be divided into three main classes: algebraic constructions, pseudo-random generation and heuristic techniques.

\subsection{Algebraic constructions}

This approach is based on S-box generation according to certain mathematical principles. The used algebraic constructions rely either on proven mathematical relations or on the construction of bigger S-boxes from smaller ones [13]. Among the first are the finite field inversion mappings, the power mappings, etc. This is the most popular approach, because S-boxes generated in such a way are known to optimize all the desired criteria. The finite field operation of inversion has been shown to achieve the best known combination of high algebraic degree, high nonlinearity and low autocorrelation [24]. For example, an $(8 \times 8)$ S-box constructed in such a way will possess algebraic degree of 7 , nonlinearity of $112, \delta$ uniformity of 4, and maximum non-zero autocorrelation of 32. The S-box of AES [10] is constructed with an inverse mapping followed by an affine transformation in the finite field. It is shown that certain algorithmic S-boxes possess complete linear redundancy and as such all component Boolean functions, except the zero one, are of the same equivalence class $[2,33]$. The equivalence class of the finite field inversion for the $(8 \times 8)$ case is described in Table 2 .

Table 2: Finite Field Inversion Equivalence Class Properties

\begin{tabular}{|l|c|}
\hline Algebraic degree: $\operatorname{deg}(S)$ & 7 \\
\hline Nonlinearity: $N_{S}$ & 112 \\
\hline Maximum absolute non-zero autocorrelation: $A C(S)_{\max }$ & 32 \\
\hline Absolute ACT Spectrum Distribution & $\{(0,32),(8,84),(16,74),(24,52),(32,13),(256,1)\}$ \\
\hline Absolute WHT Spectrum Distribution & $\{(0,17),(4,48),(8,36),(12,40)$, \\
& $(16,34),(20,24),(24,36),(28,16),(32,5)\}$ \\
\hline
\end{tabular}

Besides the simple algebraic structure of S-boxes obtained by algebraic constructions and the potential vulnerability to the algebraic attack [9], these techniques are not typically designed to produce a large set of S-boxes. 


\subsection{Pseudo-random generation}

The second approach is based on using of some pseudo-random generation or a table of random numbers to generate the entries in the S-box and then test the S-box whether is good or not. Using of this approach will take a great effort to find a good S-box because of the contradiction of the desired criteria and the small number of good S-boxes among all in the whole space, which quickly becomes infeasible as the size of the input space increases. For example, in the case of $(8 \times 8)$ S-boxes, the highest value for nonlinearity found is $98-100$ $[20,21]$, and these with nonlinearity 100 found were only four out of 50 million S-boxes generated.

\subsection{Heuristic techniques}

The heuristic techniques involve a process of iteratively improving given S-box or S-boxes with respect to one or more properties. Because they use directed search methods, unlike the algebraic constructions they are able of producing a large number of S-boxes, which are not optimal but semi-optimal, often ensure better resistance against algebraic attack and not the least make people believe in the absence of trapdoors. Specific heuristic techniques include the hill climbing method, the simulated annealing method, the genetic algorithm or a combination of these. The hill climbing method involves the application of small modifications of one or more distinct elements in order iteratively to improve one or more cryptographic properties. The highest nonlinearity achieved by this method is 100 [20]. Simulated annealing method provides an extension to the hill climbing technique in which the search process is able to move out of a local optimum in order to continue. For the case of $(8 \times 8) \mathrm{S}$-boxes by using this method S-boxes possessing nonlinearity 102 are generated [8]. The genetic algorithms work with a population of candidate solutions. Aiming to produce future populations of S-boxes possessing desired properties, they apply three operations inspired by natural evolution selection, crossover and mutation. During the selection process parental pairs, selected from an initial solution pool, interbreed to produce children. The breeding scheme is based on the mechanism of crossover like in sexual reproduction. Genetic variation is result of breaking and recombining of parent genes, thus producing offspring with combined parents attributes. The crossover mechanism operates on parent genes selected by a crossover point which is chosen randomly. Everything before this point is copied from the first parent and then everything after this point is copied from the other. After the breeding of all parental pairs when all the children are born, a mutation is applied on them with the intention of producing random changes in the offspring and thus preventing from falling into a local optimum. Then a fitness function to each of the children is applied. Based on the fitness values obtained and the solutions in the current pool a decision which of the children and which of the solutions in the current pool will replace the candidate solutions in the parent pool is made. After that a new evolutionary process begins and so on until reaching of some threshold number of iterations chosen in advance. At the end, the fittest solution of the final generation is the best solution. In [32], $(8 \times 8)$ S-boxes having nonlinearity 104 are produced by combining a special genetic algorithm and total tree searching.

\section{New Method}

The proposed new method is based on a genetic algorithm working in a reversed way. The basic idea is to start from an initial pool of S-boxes based on a finite field inversion with respect to a polynomial basis and go down from their cryptographic characteristics optimal 
values until reaching some close to the optimal and chosen in advance threshold values, which are still satisfactory. The main goal of the algorithm is a rapid construction of a variety of big (from $(8 \times 8)$ up to $(16 \times 16))$ bijective $\mathrm{S}$-boxes which possess cryptographic properties close to optimal ones, but which have more complex algebraic structure and possess zero linear redundancy. A check-up, whether the S-box nonlinearity is greater than the nonlinearity $N_{\text {inv }}$ of S-boxes based on inversion in the finite field, is done and if so, the S-box is saved in a file.

\subsection{Genetic Algorithm 1}

Genetic algorithm 1 (GA1) works in a reverse way, hence we start from S-boxes achieving the optimal known values for nonlinearity, algebraic degree and $\delta$-uniformity. The input of the algorithm is an initial parent pool which contains a number of $T$ S-boxes of dimensions $(n \times n)$. Some of the S-boxes in the initial pool are based on finite field inversion with respect to a polynomial basis and the others are results from applying affine transformations to the finite field inversion S-boxes. The algorithm makes use of three main functions:

1. The breeding function, denoted by breeding $\left(P_{i}, P_{j}, C o P_{1}, C o P_{2}, c n t\right)$, has five input arguments - two distinct parents from the parent pool $P_{i}$ and $P_{j}$, two distinct crossover points $\mathrm{CoP}_{1}$ and $\mathrm{CoP}_{2}$, which are randomly generated integers $\in\left(1,2^{n}\right)$, and a five-valued counter cnt. The two distinct crossover points $\mathrm{CoP}_{1}$ and $\mathrm{CoP}_{2}$ point out the positions where the breaking of the genes of $P_{i}$ and $P_{j}$ respectively is done. The five different values of the counter cnt specify the order (straight or reversed) in which the two parts with parents genes divided by the crossover point are copied into the children. Using this approach helps an additional element of randomness to be provided in the computational process by changing the direction of convergence which in turn enables the genetic process to deviate away from a point of local optimum. As an output the breeding function returns two children $C h_{1}$ and $C h_{2}$. The children are obtained by the following crossover scheme: All $T$ S-boxes $P_{1}, P_{2}, \ldots, P_{T}$ in the parental pool are represented by their look-up tables, each containing all $2^{n}$ S-box outputs respective to all $2^{n}$ possible lexicographically ordered inputs. Thus, as a result of the breeding of the parental pair $\left(P_{i}, P_{j}\right)$, one of all possible $\frac{T(T-1)}{2}$ pairs, $\left(C h_{1}, C h_{2}\right)$ is obtained. According to the values of the counter cnt, the way $C h_{1}$ and $C h_{2}$ are obtained is different. In the case when $c n t=1$, the rule is:

$C h_{1}\left(x_{k}\right)=P_{1}\left(x_{k}\right)$, where $x_{k} \in B$ and $k=1,2, \ldots, C o P_{1}$

$C h_{1}\left(x_{k}\right)=P_{2}\left(x_{k}\right)$, where $x_{k} \in B$ and $k=C o P_{1}+1, C o P_{1}+2, \ldots, 2^{n}$

$C h_{2}\left(x_{p}\right)=P_{2}\left(x_{p}\right)$, where $x_{p} \in B$ and $p=1,2, \ldots, C o P_{2}$

$C h_{2}\left(x_{p}\right)=P_{1}\left(x_{p}\right)$, where $x_{p} \in B$ and $p=C o P_{2}+1, C o P_{2}+2, \ldots, 2^{n}$.

When cnt $=2,3,4$ and 5, one of the two parts of one of the parents is used reversely (a kind of permutation is applied). An example for the case $(8 \times 8)$, cnt $=1, C o P_{1}=k$ and $C o P_{2}=m$, where $k, m \in(1,256)$, is given in Table 3 .

Table 3: The Crossover Mechanism Example for $c n t=1$

\begin{tabular}{|l|c|c|c|c|c|c|c|c|c|c|}
\hline Parent $P_{1}$ & $y_{1}$ & $y_{2}$ & $\ldots$ & $y_{k-1}$ & $y_{k}$ & $y_{k+1}$ & $y_{k+2}$ & $\ldots$ & $y_{255}$ & $y_{256}$ \\
\hline Parent $P_{2}$ & $v_{1}$ & $v_{2}$ & $\ldots$ & $v_{m-1}$ & $v_{m}$ & $v_{m+1}$ & $v_{m+2}$ & $\ldots$ & $v_{255}$ & $v_{256}$ \\
\hline Child $C h_{1}$ & $y_{1}$ & $y_{2}$ & $\ldots$ & $y_{k-1}$ & $y_{k}$ & $v_{k+1}$ & $v_{k+2}$ & $\ldots$ & $v_{255}$ & $v_{256}$ \\
\hline Child $C h_{2}$ & $v_{1}$ & $v_{2}$ & $\ldots$ & $v_{m-1}$ & $v_{m}$ & $y_{m+1}$ & $y_{m+2}$ & $\ldots$ & $y_{255}$ & $y_{256}$ \\
\hline
\end{tabular}


2. The mutation function, denoted by modeling $(C h)$, has as an input argument any child born in result of the breeding of any of the parental pairs. The function is applied with two main purposes. The first one comes in response to the unwanted mutation which may have occurred in the children after the crossover, namely the loss of their bijection property. So, the bijection must be repaired. And the second purpose is some additional randomness to the computational process to be added which will help in providing a chance for deviation from a local optimum similarly to the usage of the counter cnt. The output of the mutation function is a child which is a permutation. The modeling process is described bellow:

Let $\left(C h_{1}, C h_{2}\right)=\operatorname{breeding}\left(P_{i}, P_{j}, C o P_{1}, C o P_{2}, c n t\right)$ and let their Look-up tables are:

$$
\begin{aligned}
& C h_{1}=\left[y_{1}, y_{2}, \ldots, y_{C o P_{1}-1}, y_{C o P_{1}}, y_{C o P_{1}+1}, \ldots, y_{2^{n}}\right] \\
& C h_{2}=\left[v_{1}, v_{2}, \ldots, v_{C o P_{2}-1}, v_{C o P_{2}}, v_{C o P_{2}+1}, \ldots, v_{2^{n}}\right] .
\end{aligned}
$$

Clearly, each of the two parts $\left\{y_{1}, y_{2}, \ldots, y_{C o P_{1}}\right\}$ and $\left\{y_{C o P_{1}+1}, y_{C o P_{1}+2}, \ldots, y_{2^{n}}\right\}$ of $C h_{1}$, and each of the two parts $\left\{v_{1}, v_{2}, \ldots, v_{C o P_{2}}\right\}$ and $\left\{v_{C o P_{2}+1}, v_{C o P_{2}+2}, \ldots, v_{2^{n}}\right\}$ of $C h_{2}$, divided respectively by $\mathrm{CoP}_{1}$ and $\mathrm{CoP}_{2}$, consist of distinct elements because of the bijective property of the parents. So, as accurately to the order, $\left\{y_{1}, y_{2}, \ldots, y_{C o P_{1}}\right\}$ is part of $P_{i}$ and $\left\{y_{C o P_{1}+1}, y_{C o P_{1}+2}, \ldots, y_{2^{n}}\right\}$ is part of $P_{j}$, some of the elements of $\left\{y_{1}, y_{2}, \ldots, y_{C o P_{1}}\right\}$ can be repeated in $\left\{y_{C o P_{1}+1}, y_{C_{o P}+2}, \ldots, y_{2^{n}}\right\}$. The modeling of the child consists in one by one consequently checking for repetition the elements of $\left\{y_{C o P_{1}+1}, y_{C o P_{1}+2}, \ldots, y_{2^{n}}\right\}$ in $\left\{y_{1}, y_{2}, \ldots, y_{C o P_{1}}\right\}$. If so, a new element is repeatedly randomly generated, and if it is not met yet, it replaces the duplicate. The procedure is applied for both of the children. At the end, they are permutations.

3. The fitness function, denoted by fitness $(C h)$, has as an input argument any child, which has already been modeled, and returns a value playing the role of a measure taken to ascertain whether the child will survive to the new generation or not. The fitness value is the nonlinearity value of the child $N_{c h}$. The fitness test is passed by the child $C h$ if $N_{C h} \geq N_{t h r}$, where $N_{t h r}$ is the threshold nonlinearity value chosen in advance. If $N_{C h}>N_{t h r}$, then the child takes its place in the offspring pool. If $N_{C h}=N_{t h r}$, then besides the child takes its place in the offspring pool, it is also saved in a file. If $N_{C h}>N_{i n v}$, then the child is saved in a file. Otherwise, the child does not survive to the next generation and it is left off. If after the breeding of all pairs from the parent pool the offspring pool is not totally full, the breeding process starts all over again with the pair $\left(P_{1}, P_{2}\right)$. This makes sense, because the crossover points $\mathrm{CoP}_{1}$ and $\mathrm{CoP}_{2}$ are randomly chosen which ensures that the next pair of children of $P_{1}$ and $P_{2}$ will be different from the previous one. When the offspring pool gets full of children, if all of them have nonlinearities equal to the threshold value $N_{t h r}$, the algorithm stops. Otherwise the children replace their parents in the parent pool and so on to the next generation.

\subsection{Genetic Algorithm 2}

The Genetic algorithm 2 (GA2) provided in this section is a slight modification of GA1 in which an additional cost function is applied together with the fitness function in order to help deciding whether the respective child will survive to the next generation or not. The cost function is based on the WHT spectrum and is taken from the family of functions in [8]:

$$
\operatorname{cost}(S)=\sum_{c=\left(c_{1}, c_{2}, \ldots, c_{m}\right) \in \mathbb{B}^{m} \backslash\{0\}} \sum_{\omega \in \mathbb{B}^{n}}\left|\widehat{F}_{c_{1} f_{1} \oplus c_{2} f_{2} \oplus \ldots \oplus c_{m} f_{m}}(\omega)-X\right|^{R},
$$


where $X$ and $R$ are real-valued parameters and $\widehat{F}_{c_{1} f_{1} \oplus c_{2} f_{2} \oplus \ldots \oplus c_{m} f_{m}}(\omega)$ is the WHT of the component Boolean function $c_{1} f_{1} \oplus c_{2} f_{2} \oplus \ldots \oplus c_{m} f_{m}$ of the S-box given by $c$. More precisely, GA2 uses the specific cost function for $m=n$ with $(X, R)=(21,7)$, as proposed in [32].

The main difference between the two genetic algorithms proposed is that in addition to GA1 in GA2 a child, which has already been modeled, passes the fitness test not only in the case when its fitness value is good enough, i.e. $N_{C h} \geq N_{t h r}$, but also when in addition its cost value is smaller than the one of its parent, i.e. $\operatorname{cost}\left(C h_{1}\right)<\operatorname{cost}\left(P_{1}\right)$. The pseudo-codes of both algorithms are presented in Appendix A.

\section{Experimental Results}

In this section the results of generating a set of $T(n \times n)$ bijective S-boxes using the two variants of genetic algorithms are provided.

\subsection{Results Obtained with $G A 1$}

1) The case $\boldsymbol{n}=\mathbf{8}$ The results obtained by GA1 are closely related to the linear redundancy property. If it is considered as a vital one, the output of the GA1 are S-boxes which possess zero linear redundancy, but their nonlinearities are at most 108. Otherwise, S-boxes with nonlinearity 110 and 112 are achieved, but they possess some linear redundancy.

The properties of representatives obtained with $G A 1$ for the case $n=8$ with an initial pool full of 200 S-boxes and $N_{t h r}$ equal to 104,106 and 108 are provided in Table 4.

Table 4: S-boxes generated with $G A 1$ in the case $n=8$ and $N_{t h r}=104,106$ and 108

\begin{tabular}{|l|c|c|c|c|c|c|c|c|}
\hline S-BOX & $N_{t h r}$ & $N_{S}$ & $\operatorname{deg}(S)$ & $A C(S)_{\max }$ & $\delta$-uniformity & Fixed points & Linear Redundancy \\
\hline AES & S-BOX & - & 112 & 7 & 32 & 4 & 2 & complete \\
\hline GA1 & S-BOX 1 & 104 & 104 & 7 & 64 & 6 & 2 & zero \\
\hline GA1 S-BOX 2 & 106 & 106 & 6 & 56 & 6 & 2 & zero \\
\hline GA1 & S-BOX 3 & 108 & 108 & 6 & 48 & 6 & 0 & zero \\
\hline
\end{tabular}

The respective cryptographic properties distributions of the S-box component Boolean functions are provided in Table 5, where the pair $\left(\right.$ value $_{1}$, value $\left._{2}\right)$ means that the respective

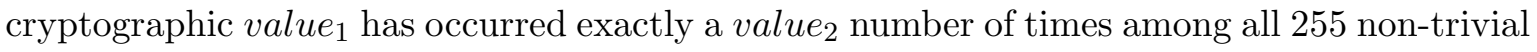
S-box component Boolean functions.

Table 5: Distributions of the component Boolean functions of $G A 1$ S-boxes in the case $n=8$

\begin{tabular}{|ll|c|c|c|c|}
\hline LCBFs distributions & $N_{L C B F s}$ & $\operatorname{deg}(L C B F s)$ & $A C_{\max }(L C B F s)$ & Equivalence classes \\
\hline AES & S-BOX & $(112,255)$ & $(7,255)$ & $(32,255)$ & 1 \\
\hline GA1 & S-BOX 1 & $(112,14)(110,116)$ & $(7,255)$ & $(64,6)(56,54)$ & 255 \\
& & $(108,92)(106,29)$ & & $(48,141)(40,54)$ & \\
\hline GA1 & S-BOX 2 & $(104,4)$ & & & \\
& & $(12,60)(110,160)$ & $(7,254)$ & $(56,2)(48,63)$ & 255 \\
\hline GA1 & S-BOX 3 & $(112,99)(110,149)$ & $(6,1)$ & $(40,180)(32,10)$ & \\
& $(108,7)$ & $(6,1)$ & $(48,14)(40,202)$ & 255 \\
& \multicolumn{2}{|c|}{$(32,39)$} & \\
\hline
\end{tabular}


As it can be seen from Tables 4 and 5, the obtained S-boxes possess zero linear redundancy, that is all non-trivial 255 component Boolean functions belong to distinct equivalence classes. This is an improvement in terms of linear redundancy compared to the inversion S-boxes where all 255 component Boolean functions are from the same equivalence class (Table 2) and hence a complete linear redundancy is available. The particular S-boxes are presented in Appendix B in hexadecimal notation.

2) The case $\boldsymbol{n}=\mathbf{1 6}$ The properties of representatives for $n=16$ with an initial pool full of 50 S-boxes and $N_{t h r}$ equal to 32400,32428 and 32476 are provided in Table 6.

Table 6: S-boxes generated with $G A 1$ in the case $n=16$ and $N_{t h r}=32400,32428$ and 32476

\begin{tabular}{|c|c|c|c|c|}
\hline BOX & $N_{t h r}$ & $N_{S}$ & $\operatorname{deg}(S)$ & $A C(S)_{\max }$ \\
\hline Inversion S-BOX & - & 32512 & 15 & 512 \\
\hline GA1 S-BOX 1 & 32400 & 32400 & 15 & 976 \\
\hline GA1 $\quad$ S-BOX 2 & 32428 & 32428 & 15 & 864 \\
\hline S-BOX 3 & 32476 & 32476 & 14 & 616 \\
\hline
\end{tabular}

\subsection{Results Obtained with GA2}

GA2 is a little slower than GA1. Furthermore, it should be noted that its results are better only in terms of linear redundancy. S-boxes with nonlinearity 110 and zero linear redundancy were obtained. One reason could be the additional cost function in the fitness which slows up the offspring obtaining and thus inserts more randomness in the process. The other one could be that in all tests with GA2, different paths have been selected because of the randomness inserted in the breeding and the mutation step. The properties of representatives obtained with GA2 for $n=8$ with an initial pool of 200 S-boxes and $N_{t h r}$ equal to 106, 110 and 112 are provided in Table 7 . The distributions of the S-box component functions are provided in Table 8. Similar to GA1, the improvement in terms of zero linear redundancy can also be observed, excluding the case $N_{t r h}=112$, where only 147 out of 255 distinct equivalence classes were achieved. The particular S-boxes can be viewed in Appendix C.

We should note that with GA1 and GA2 we found no S-boxes satisfying $N_{C h}>N_{i n v}$.

Table 7: S-boxes generated with GA2 in the case $n=8$ and $N_{t h r}=106,110$ and 112

\begin{tabular}{|c|c|c|c|c|c|c|c|}
\hline S-BOX & $N_{t h r}$ & $N_{S}$ & $\operatorname{deg}(S)$ & $A C(S)_{\max }$ & $\delta$-uniformity & Fixed points & Linear Redundancy \\
\hline AES S-BOX & - & 112 & 7 & 32 & 4 & 2 & complete \\
\hline GA2 S-BOX 1 & 106 & 106 & 6 & 48 & 6 & 0 & zero \\
\hline GA2 S-BOX 2 & 110 & 110 & 7 & 40 & 6 & 0 & zero \\
\hline GA2 S-BOX 3 & 112 & 112 & 7 & 32 & 6 & 0 & some (147 of 255 ) \\
\hline
\end{tabular}

The proposed method is fast enough in repeatedly producing of thousands of $(8 \times 8) \mathrm{S}$ boxes with nonlinearity up to 112. Together with the high nonlinearity, most of the S-boxes possess properties which are close to the finite filed inversion-based ones. At a reasonable price of small deviations from the best known values, more complex algebraic structure and zero linear redundancy is achieved except for $N_{t h r}=112$, where the maximum number of different equivalence classes for all component Boolean functions found was 147. Even for $n=16$ the 
Table 8: Distributions of the component Boolean functions of GA2 S-boxes in the case $n=8$

\begin{tabular}{|c|c|c|c|c|}
\hline$L C B F s$ distributions & $N_{L C B F s}$ & $\operatorname{deg}(L C B F s)$ & $A C_{\max }(L C B F s)$ & Equivalence classes \\
\hline AES S-BOX & $(112,255)$ & $(7,255)$ & $(32,255$ & 1 \\
\hline GA2 S-BOX 1 & $\begin{array}{ll}(112,85) & (110,150) \\
(108,18) & (106,102)\end{array}$ & $\begin{array}{c}(7,254) \\
(6,1)\end{array}$ & $\begin{array}{c}(48,46)(40,160) \\
(32,49)\end{array}$ & 255 \\
\hline GA2 S-BOX 2 & $\begin{array}{c}(114,1)(112,162) \\
(110,92)\end{array}$ & $(7,255)$ & $(40,122)(32,133)$ & 255 \\
\hline GA2 S-BOX 3 & $(112,255)$ & $(7,255)$ & $(32,255)$ & 147 \\
\hline
\end{tabular}

algorithm is relatively fast if $N_{t h r}$ is chosen to be close to $N_{i n v}=32512$. It has found 50 S-boxes in 3 and a half months. We can obtain S-boxes of each possible nonlinearity and the closer to $N_{i n v}$ the faster the algorithm is. Considering the huge time and space resources needed for the computations when generating $(16 \times 16)$ S-boxes, the proposed method proves to be more applicable than any of the known pseudo-random and heuristic techniques. 


\section{References}

1. E. Biham. On Matsui's linear cryptanalysis. In Eurocrypt'94, volume 950 of LNCS, pages 341-355. Springer, 1994.

2. E. Biham. Observations on the relations between bit-functions of many s-boxes. In The 3rd NESSIE conference, November 2002.

3. E. Biham and A. Shamir. Differential cryptanalysis of des-like cryptosystems. In Advances in Cryptology CRYPTO'90, volume 537 of LNCS, pages 2-21. Springer Verlag, 1991.

4. E. Biham and A. Shamir. Differential cryptanalysis of des-like cryptosystems. Journal of Cryptology, 4:3-72, 1991.

5. C. Carlet. Boolean Models and Methods in Mathematics, Computer Science, and Engineering, chapter Boolean Functions for Cryptography and Error Correcting Codes, pages 257-397. Cambridge University Press, 2010.

6. C. Carlet. Boolean Models and Methods in Mathematics, Computer Science, and Engineering, chapter Vectorial Boolean Functions for Cryptography, pages 257-397. Cambridge University Press, 2010.

7. F. Chabaud and S. Vaudenay. Links between differential and linear cryptanalysis. In Advances in Cryptology EUROCRYPT'94, volume 950 of LNCS, pages 356-365. Springer Verlag, 1995.

8. J.A. Clark, J.L. Jacob, and S. Stepney. The design of s-boxes by simulated annealing. New Generation Computing Archive, 23(3), September 2005.

9. N. T. Courtois and J. Pieprzyk. Cryptanalysis of block ciphers with overdefined systems of equations. In Advances in Cryptology - ASIACRYPT'02, volume 2501 of LNCS, pages 267-287. Springer Verlag, 2002.

10. J. Daeman and V. Rijmen. The design of Rijndael: AES The advanced Encryption Standard. Springer Verlag, 2002.

11. J. Daemen, R. Govaerts, and J. Vandewalle. Correlation matrices. In FSE'94, volume 1008 of LNCS, pages 275-285. Springer, 1995.

12. J. Fuller and W. Millan. Linear redundancy in s-boxes. In FSE'03, volume 2887 of LNCS, pages 74-86. Springer, 2003.

13. B. Gerard, V. Grosso, M. Naya-Plasencia, and F.-X. Standaert. Block ciphers that are easier to mask: How far can we go? In CHES'03, volume 8086 of LNCS, pages 383-399. Springer, 2003.

14. J. Dj. Golić. Fast low order approximation of cryptographic functions. In Advances in Cryptology EUROCRYPT'96, volume 1070 of LNCS, pages 268-282. Springer Verlag, 1996.

15. O. Kazymyrov, V. Kazymyrova, and R. Oliynykov. A method for generation of high-nonlinear s-boxes based on gradient descent. IACR Cryptology ePrint Archive (2013), http://eprint.iacr.org/2013/578.

16. L.Goubin, A.Martinelli, and M.Walle. Impact of s-boxes size upon side channel resistance and block cipher design. In AFRICACRYPT'13, volume 7918 of LNCS, pages 240-259. Springer, 2013.

17. M. Matsui. Linear cryptanalysis method for DES cipher. In Advances in Cryptology EUROCRYPT'93, volume 765 of LNCS, pages 386-397. Springer Verlag, 1994.

18. F. J. McWilliams and N.J.A. Sloane. The Theory of Error-Correcting Codes. North-Holland, 1978.

19. W. Meier and O. Staffelbach. Nonlinearity criteria for cryptographic functions. In Advances in Cryptology EUROCRYPT'89, volume 434 of LNCS, pages 549-562. Springer Verlag, 1990.

20. W. Millan. How to improve the nonlinearity of bijective s-boxes. In Australian Conference on Information Security and Privacy 1998, volume 1438, pages 181-192. Springer Verlag, 1998.

21. W. Millan, L. Burnett, G. Carter, A. Clark, and E. Dawson. Evolutionary heuristics for finding cryptographically strong s-boxes. In ICICS'99, volume 1726 of LNCS, pages 263-274. Springer, 1999.

22. W. L. Millan. Low order approximation of cipher functions. In Cryptography: Policy and Algorithms Conference, Proceedings, volume 1029 of LNCS, pages 144-155. Springer Verlag, 1996.

23. K. Nyberg. Perfect nonlinear s-boxes. In Advances in Cryptology EUROCRYPT'91, volume 547 of LNCS, pages 378-386. Springer Verlag, 1992.

24. K. Nyberg. Differentially uniform mappings for cryptography. In Advances in Cryptology EUROCRYPT'93, volume 765 of LNCS, pages 55-64. Springer Verlag, 1994.

25. B. Preneel. Analysis and Design of Cryptographic Hash Functions. PhD thesis, KU Leuven, 1994.

26. L. Qu, Y. Tan, C. Li, and G. Gong. More constructions of differentially 4-uniform permutations on $\mathbb{F}_{2^{2 k}}$. In arxiv.org/pdf/1309.7423, 2013.

27. L. Qu, Y. Tan, C. Tan, and C. Li. Constructing differentially 4-uniform permutations over $\mathbb{F}_{2^{2 k}}$ via the switching method. IEEE Transactions on Inform. Theory, 59(7):4675-4686, 2013.

28. O. S. Rothaus. On bent functions. Journal of Combinatorial Theory, 20(3):300-305, May 1976.

29. J. Seberry, X. M. Zhang, and Y. Zheng. Systematic generation of cryptographically robust s-boxes. In Proceedings of the first ACM Conference on Computer and Communications Security, pages 171-182. The Association for Computing Machinery, Fairfax, VA, 1993. 
30. J. Seberry, X. M. Zhang, and Y. Zheng. Relationships among nonlinearity criteria. In Advances in Cryptology - EUROCRYPT'94, volume 950 of LNCS, pages 376-388. Springer Verlag, 1995.

31. C. E. Shannon. Communication theory of secrecy systems. Bell System Technical Journal, 28:656-715, 1949.

32. P. Tesař. A new method for generating high non-linearity s-boxes. Radioengineering, 19(1):23-26, 2010.

33. A.M. Youssef and S.E. Tavares. On some algebraic structures in the aes round function. Technical Report 2002/144, Cryptology ePrint Archive, 2002.

34. X. Zhang, Y. Zheng, and H. Imai. Relating differential distribution tables to other properties of substitution boxes. Designs, Codes and Cryptography, 19:45-63, 2000. 


\section{A Appendix A}

\section{A.1 Genetic Algorithm 1 pseudo code}

1. Step 1 (Initializing step)

In this step the parameters of the algorithm are defined:

- Define an integer $n$ representing the dimension of the $(n \times n)$ S-box.

- Define an integer $T$ representing the number of the S-boxes in the parent pool $(P P)$.

- Define an even integer $N_{t h r} \leq N_{i n v}$ representing the nonlinearity threshold value.

- Generate a number of $T(n \times n)$ S-boxes and put them in the $(P P)$. Some based on inversion in the finite field $G F\left[2^{n}\right]$ and some obtained in result of application of affine transformations to the generated algorithmic S-boxes.

- Create an empty offspring pool $(O P)$ of size $T$.

- Set the counter cnt value to be 0 .

- Set the parents indexes $t$ and $r$ to be 1 .

2. Step 2 (Breeding step)

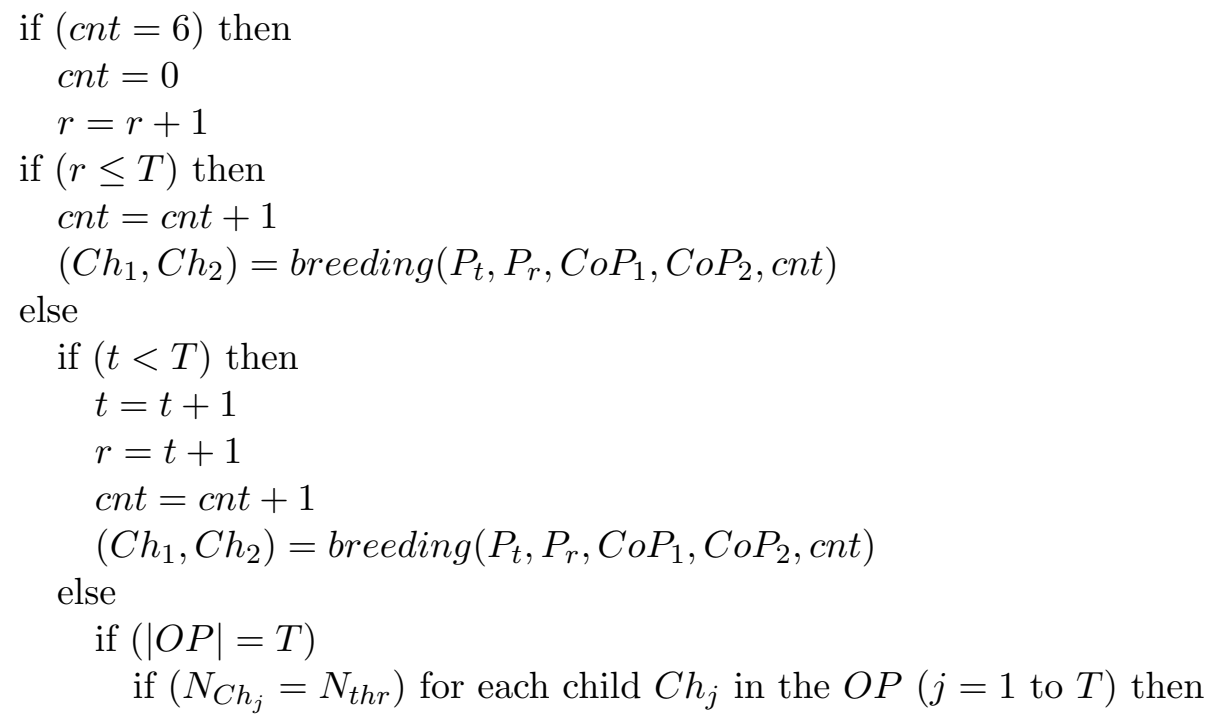

STOP the algorithm go to Step 5

else

move and replace $O P$ into $P P$

cnt $=0$

$t=1$

$r=1$

go to Step 2

else

cnt $=0$

$t=1$

$r=1$

go to Step 2

go to Step 3

3. Step 3 (Mutation step)

$C h_{1}=$ modeling $\left(C h_{1}\right)$ and $C h_{2}=\operatorname{modeling}\left(C h_{2}\right)$

go to Step 4 
4. Step 4 (Fitness step)

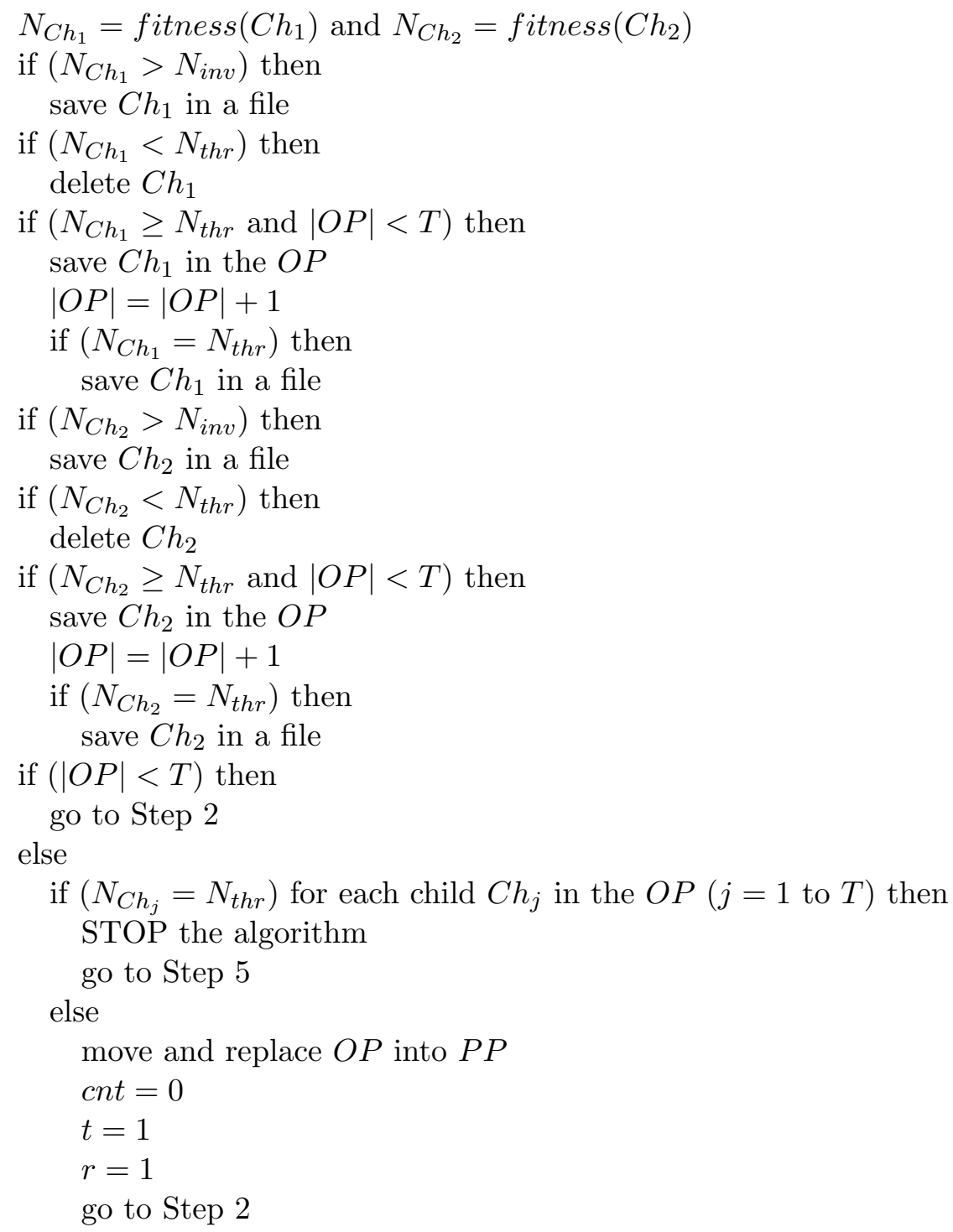

5. Step 5 (Solution pool)

In the offspring pool $O P$ are the all desired $T$ S-boxes with $N=N_{t h r}$.

\section{A.2 Genetic Algorithm 2 pseudo code}

The pseudo code of GA2 is exactly the same as the pseudo code of GA1 with a few exceptions.

Only lines 6 and 15 of $G A 1$ fitness step, namely

"if $\left(N_{C h_{1}} \geq N_{t h r}\right.$ and $\left.|O P|<T\right)$ then" and

"if $\left(N_{C h_{2}} \geq N_{t h r}\right.$ and $\left.|O P|<T\right)$ then",

are replaced with

"if $\left(N_{C h_{1}} \geq N_{t h r}\right.$ and $C_{C h_{1}}<C_{P_{1}}$ and $\left.|O P|<T\right)$ then" and

"if $\left(N_{C h_{2}} \geq N_{t h r}\right.$ and $C_{C h_{2}}<C_{P_{2}}$ and $\left.|O P|<T\right)$ then" respectively,

where $C_{P_{1}}=\operatorname{cost}\left(P_{1}\right), C_{P_{2}}=\operatorname{cost}\left(P_{2}\right), C_{C h_{1}}=\operatorname{cost}\left(C h_{1}\right)$ and $C_{C h_{2}}=\operatorname{cost}\left(C h_{2}\right)$. 


\section{B S-boxes generated with $G A 1$}

\section{B.1 S-box No $1\left(N_{S}=104, \operatorname{deg}(S)=7, A C(S)_{\max }=64, \delta=6\right)$}

$\{0 x 520 x 53$ 0xDF 0xA4 0x99 0x00 0x29 0x83 0xBA 0x1D 0x7B $0 x 92 \quad 0 x E 2 \quad 0 x B 3$ 0xB7 $0 x 95$ $0 x 26$ 0xE6 0xF8 0x19 0xCB 0x79 0x32 0x0D 0x0A 0x6D 0xAF $0 x 9 E$ 0xAD 0x12 0xBC 0xE0

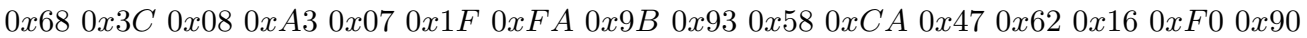

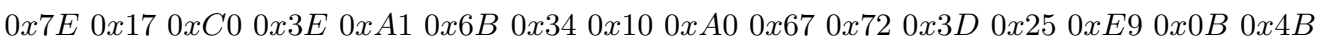
$0 x 4 F \quad 0 x A C$ 0x65 $0 x 350 x 7 F \quad 0 x 630 x A 7 \quad 0 x 3 B \quad 0 x F 50 x 36 \quad 0 x F 90 x 410 x 06 \quad 0 x 770 x B B \quad 0 x 5 B$ 0xBF 0x0E $0 x 57$ 0x98 0x1E 0x76 0xD5 0xED 0x4A 0x6C 0x70 0xA2 $0 x 03$ 0xBE $0 x 33$ 0x45

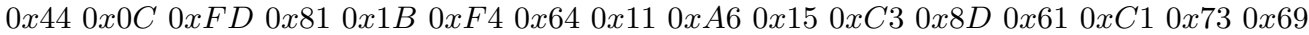
$\begin{array}{lllllllllllllllll}0 x 2 B & 0 x E 5 & 0 x C 5 & 0 x D 7 & 0 x 42 & 0 x E 7 & 0 x E 8 & 0 x 6 E & 0 x E 4 & 0 x 22 & 0 x 82 & 0 x 54 & 0 x F 3 & 0 x A 8 & 0 x D 3 & 0 x D 0\end{array}$ 0xD1 0x2C 0x2D 0xD2 0xC4 0x21 0xEC 0x04 0xC9 0xCC 0xC7 0x8B 0xA5 0x50 0xEB 0xF6 $\begin{array}{llllllllllllllllll}0 x 8 C & 0 x 38 & 0 x 60 & 0 x 3 F & 0 x 8 A & 0 x D 8 & 0 x D 6 & 0 x 20 & 0 x 78 & 0 x 46 & 0 x C D & 0 x D A & 0 x A B & 0 x 8 E & 0 x D B & 0 x C 8\end{array}$ 0xA9 0x2E 0x7C 0x91 0xDD 0xEA 0x37 0x1A 0x74 0x9A $0 x 40$ 0x18 0x9C 0xB5 $0 x 80$ 0x30 0x5E $0 x B 2 \quad 0 x 4 D$ 0xBD $0 x 43$ 0x27 0x2A $0 x 23$ oxF7 $0 x D C$ 0x24 0x6F 0xEF 0xEE $0 x D 4$ 0x05 0x59 0x7A 0x7D 0xF1 0x88 0x86 0xB6 0x5D 0xFB $0 x 75$ 0x01 0x56 0x49 0xAE $0 x F E$ 0xB4 0x28 0x55 0xFC 0x31 0x97 0x89 0xB0 0xB8 0xC6 0xD9 0x96 0x87 0xCF 0xAA $0 x C 2 \quad 0 x 39$ 0xE3 $0 x 5 F \quad 0 x 840 x B 90 x 94 \quad 0 x 5 C$ 0x9D $0 x F F \quad 0 x 5 A$ ox $1 C$ 0x85 $0 x B 1 \quad 0 x 0 F \quad 0 x 02 \quad 0 x 4 C$ 0xF2

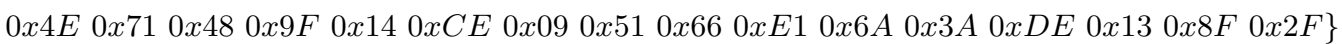

\section{B.2 S-box No $2\left(N_{S}=106, \operatorname{deg}(S)=6, A C(S)_{\max }=56, \delta=6\right)$}

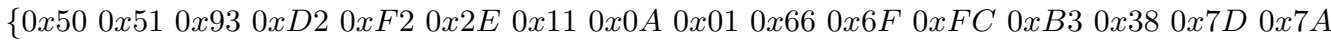
$\begin{array}{llllllllllllllllll}0 x B B & 0 x C B & 0 x 4 B & 0 x 65 & 0 x 8 C & 0 x 4 E & 0 x 06 & 0 x F 5 & 0 x E 2 & 0 x 24 & 0 x 64 & 0 x 42 & 0 x 85 & 0 x 34 & 0 x 45 & 0 x 8 D\end{array}$

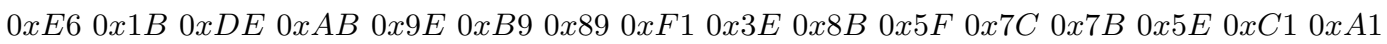
$0 x 090 x 87$ 0x6A $0 x A 4$ 0x4A $0 x 43$ 0x59 0x00 0xF9 0x33 0x62 0xA5 0x99 0x9C 0xFD $0 x 5 A$ 0x0B 0x56 0xB6 0xA7 0x17 0xEF 0xEE 0x14 0x37 0x2B 0xE7 0x71 0xFF 0x03 0xC3 0xAF

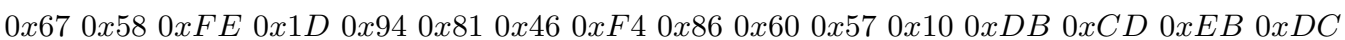
0xBF 0xD1 0xF8 $0 x 69$ 0x4D $0 x 84$ 0x2A $0 x 18$ 0x5D $0 x B 2$ 0x9A $0 x E 0 \quad 0 x 97$ 0x8E $0 x 78$ 0x8A 0xC7 0x82 0xA2 0xD4 0x49 0xE3 0xE9 0xD7 0xF7 0xB4 0x36 0x19 0xC5 0xC9 0x55 0xF3 0xBE $0 x 31$ 0x53 0x92 0x23 0xA3 0xE8 0x27 0xB0 0xA8 0xCC 0x0C 0x0F 0xEA $0 x 72$ 0xAA $0 x A 0$ 0x7E 0xAE 0x1E 0xC8 0x2C 0x83 0x20 0xC4 0x2D 0xBA 0x41 0xDA 0x0D 0xEC 0xBC $0 x 880 x 770 x 540 x 2 F \quad 0 x 07$ 0x47 0xB5 0x28 0x32 0x68 0xFB $0 x F A$ 0x5B 0x6E $0 x 020 x 1 C$ 0x3B 0x9B $0 x 48$ 0x25 0x90 0xAD 0x70 0x1A 0xD6 0x26 0xDD 0x0E 0xCE 0xBD $0 x 16$ 0x15 0xE4 0xAC 0xD3 0x52 0x04 0x80 0x8F 0x3C 0x9D 0x6C $0 x 3 A$ oxE1 $0 x 6 D$ 0x98 $0 x 74$ 0xB8 0x95 0x05 0x21 0xC6 0x35 0x4C 0x08 0x61 0xF0 0x76 0x3F $0 x 79$ 0x44 $0 x 4 F \quad 0 x 3 D$ 0x96 0xD8 0xA9 0x39 0x5C 0x29 0xF6 $0 x 12$ 0xA6 0x9F $0 x 75$ 0xCA $0 x 40$ 0xCF 0xED 0xD0 $0 x 30$ 0xC0 0x7F 0x22 0xD5 0x63 0x6B 0xB7 $0 x 13$ 0xC2 $0 x D F$ 0xE5 $0 x 73$ 0xB1 $0 x 910 x 1 F \quad 0 x D 9\}$

\section{B.3 S-box No $3\left(N_{S}=108, \operatorname{deg}(S)=6, A C(S)_{\max }=48, \delta=6\right)$}

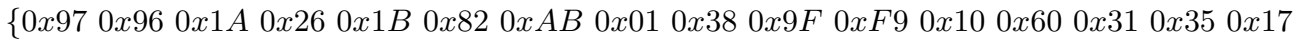
$0 x A 40 x 640 x 7 A$ 0x9B $0 x 49$ 0xFB 0xB0 0x8F $0 x 88$ 0xEF $0 x 2 D$ 0x1C 0x2F $0 x 90$ 0x3E $0 x 62$

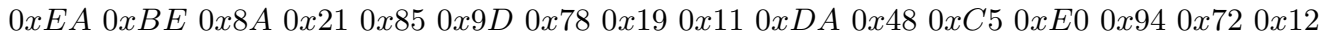

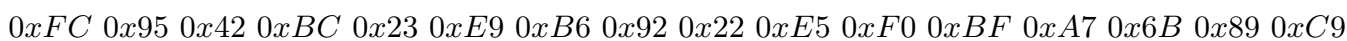
0xCD 0x2E 0xE7 0xB7 0xFD 0xE1 0x25 0xB9 $0 x 77$ 0xB4 $0 x 7 B$ 0xC3 $0 x 84$ 0xF5 $0 x 39$ 0xD9 $0 x 3 D$ 0x8C 0xD5 0xD1 0x9C 0xF4 0x57 0x6F 0xC8 0xEE $0 x F 2 \quad 0 x 200 x 810 x 3 C$ 0xB1 $0 x C 7$ 0xC6 0x8E 0x7F 0x03 0x99 0x76 0xE6 0x93 0x24 0x5D 0x41 0x0F 0xE3 0x43 0xF1 0xEB

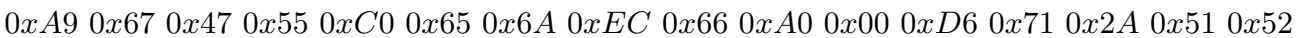
0x53 0xAE 0xAF 0x50 0x46 0xA3 0x6E $0 x 86 \quad 0 x 4 B$ 0x4E $0 x 450 x 090 x 27 \quad 0 x D 2 \quad 0 x 690 x 74$ $0 x 0 E$ 0xBA 0xE2 0xBD 0x08 0x5A 0x54 0xA2 0xFA $0 x C 4$ 0x4F $0 x 58$ 0x29 0x0C $0 x 59$ 0x4A $0 x 2 B$ 0xAC $0 x F E$ 0x13 0x5F 0x68 0xB5 0x98 0xF6 0x18 0xC2 0x9A 0x1E 0x37 $0 x 02$ 0xB2 $0 x D C$ 0x30 0xCF 0x3F 0xC1 0xA5 $0 x A 8$ 0xA1 $0 x 75$ 0x5E $0 x A 6$ 0xED $0 x 6 D$ 0x6C $0 x 56$ 0x87

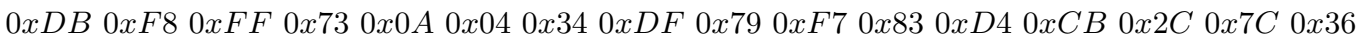

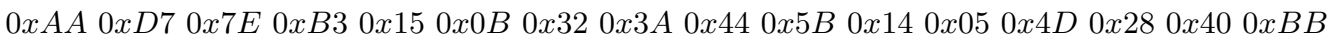
0x61 0xDD 0x06 0x3B 0x16 0xDE 0x1F 0x7D 0xD8 0x9E $0 x 07$ 0x33 0x8D $0 x 80$ 0xCE $0 x 63$ $0 x 8 B$ 0xF3 0xE8 0xE4 0xB8 0xD0 0xD3 0x5C 0x0D 0x4C 0xAD 0x70 0x1D 0xCA $0 x 910 x C C\}$ 


\section{S-boxes generated with $G A \mathscr{2}$}

\section{C.1 S-box No $1\left(N_{S}=106, \operatorname{deg}(S)=6, A C(S)_{\max }=48, \delta=6\right)$}

$\left\{\begin{array}{lllllllllllllllllll}0 x 82 & 0 x D 3 & 0 x 21 & 0 x 1 F & 0 x 95 & 0 x D C & 0 x 4 E & 0 x 86 & 0 x 5 A & 0 x 68 & 0 x 8 D & 0 x 47 & 0 x C 4 & 0 x 31 & 0 x A 0 & 0 x 5 E\end{array}\right.$

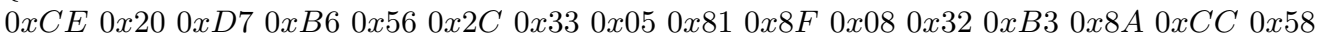
$0 x 840 x 22 \quad 0 x F 3 \quad 0 x 5 C \quad 0 x 7 B \quad 0 x 1 E \quad 0 x B 8 \quad 0 x 6 C \quad 0 x C 80 x 71 \quad 0 x F 50 x 6 F \quad 0 x 090 x 040 x 12 \quad 0 x C 5$ $0 x 50$ 0xD4 $0 x 57$ 0x0A $0 x E 7$ 0x78 $0 x F A$ 0x4D $0 x 49$ 0xB4 $0 x A 6 \quad 0 x 97 \quad 0 x 850 x 3 E$ 0xCF $0 x 0 E$ 0xA1 0x10 0xF2 0x3C 0x69 0x17 0xCD 0x00 0x2D 0x0B 0xA2 0xDB 0xBF 0x67 0xD5 0x2F $0 x 870 x 19$ 0x28 0xFB $0 x 6 A$ 0xB1 $0 x 27$ 0xB5 0x14 0x8C 0xE1 0xD9 0xEA 0x9C 0x72 $0 x 9 F$ 0xCB 0xEE 0x89 0xA9 0x3B 0x83 0xE6 0x2A 0x63 0x93 0xDF 0xC1 0x9E $0 x 41$ 0x36 0xC9 $0 x 34$ 0xF4 0xB9 0x38 0xB0 0x4B $0 x 5 B \quad 0 x 16 \quad 0 x 52 \quad 0 x B E$ 0xFC $0 x 98 \quad 0 x 77$ 0x92 $0 x E 4 \quad 0 x A B$

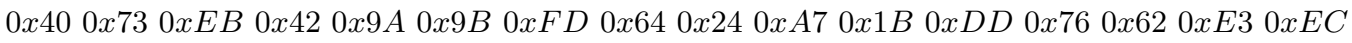
$0 x 060 x 800 x 150 x 460 x B 2 \quad 0 x 02$ 0x7D $0 x A 8$ 0x4F $0 x 18$ 0x23 0x3F 0x7A $0 x 3 A$ 0x07 $0 x 8 E$

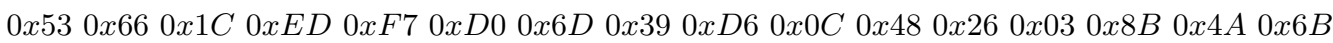

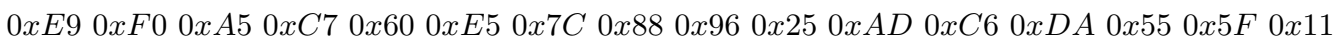
$0 x 750 x C 0 \quad 0 x 94 \quad 0 x 1 A$ 0x54 0xA3 0x44 0xE0 0x0D 0xB7 0x51 0x2E $0 x 90$ 0xE2 $0 x F 6$ 0xBA 0xBD 0x0F 0x59 0x01 0x7F 0xEF 0x70 0x37 0xAC 0xA4 0x30 0x13 0xF8 0xFE $0 x 74$ 0xDE 0xF9 0xC2 0x99 0x65 0x4C 0x29 0xFF 0xC3 0xBB 0xD1 0x35 0x6E 0x3D 0x5D 0xE8 0xAE

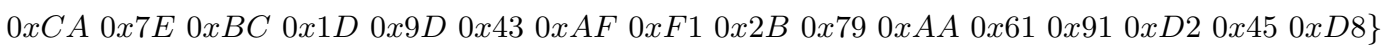

\section{C.2 S-box No $2\left(N_{S}=110, \operatorname{deg}(S)=7, A C(S)_{\max }=40, \delta=6\right)$}

$\{0 x 1 D \quad 0 x 2 B \quad 0 x D 90 x 880 x A 0 \quad 0 x E 9 \quad 0 x 7 B$ 0xB3 $0 x 6 F \quad 0 x 5 D \quad 0 x B 80 x 72 \quad 0 x F 10 x 040 x 950 x 6 B$

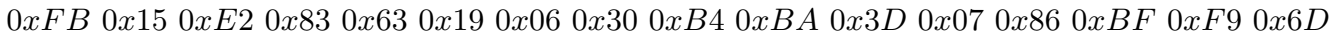
0xB1 0x17 0xC6 0x69 0x4E 0xB7 0x8D 0x59 0xFD 0x44 0xC0 0x5A $0 x 3 C$ 0x31 $0 x 27$ 0xF0 0x65 0xE1 0x62 0x3F 0xD2 0x4D 0xCF 0x78 0x7C 0x81 0x93 0xA2 0xB0 0x0B $0 x F A$ ox $3 B$ $0 x 940 x 250 x C 70 x 090 x 5 C$ 0x22 $0 x F 8$ 0x35 0x18 0x3E $0 x 97 \quad 0 x E E$ 0x8A $0 x 52 \quad 0 x E 0 \quad 0 x 1 A$ 0xB2 0x2C 0xD5 0xCE 0x5F 0x84 0x12 0x80 0x21 0xB9 0xD4 0xEC 0xDF 0xA9 0x47 0xAA

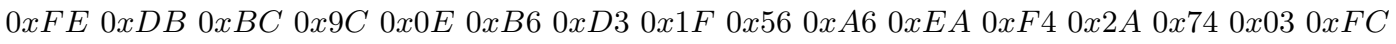
0x01 0xC1 0x8C 0x0D 0x85 0x7E $0 x 6 E$ 0x23 $0 x 67$ 0x8B $0 x C 9$ 0xAD $0 x 42$ 0xA7 $0 x D 10 x 9 E$ $0 x 750 x 46 \quad 0 x D E \quad 0 x 77 \quad 0 x A F \quad 0 x A E \quad 0 x C 8 \quad 0 x 51 \quad 0 x 11 \quad 0 x 92 \quad 0 x 2 E \quad 0 x E 8 \quad 0 x 43 \quad 0 x 57$ 0xD6 $0 x E 6$

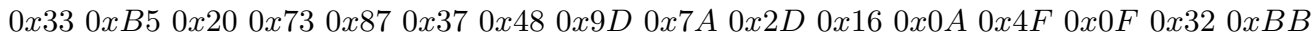
0x66 0x53 0x29 0xD8 0xC2 0xE5 0x58 0x0C 0xE3 0x39 0x7D 0x13 0x36 0xBE 0x7F 0x5E 0xDC 0xC5 0x90 0xF2 0x55 0xD0 0x49 0xBD 0xA3 0x10 0x98 0xF3 0xEF 0x60 0x6A 0x24

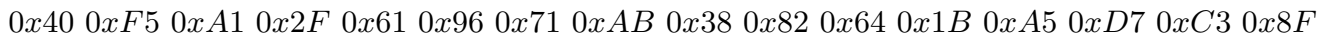
$0 x 140 x 3 A$ 0x6C $0 x 34$ 0x4A 0xDA $0 x 45$ 0x02 0x99 0x91 0x05 0x26 0xCD 0xCB $0 x 41$ 0xEB

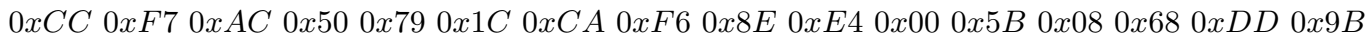

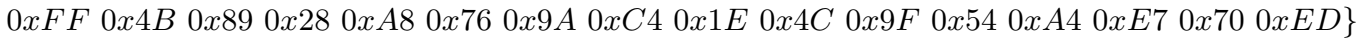

\section{C.3 S-box No $3\left(N_{S}=112, \operatorname{deg}(S)=7, A C(S)_{\max }=32, \delta=6\right)$}

$\{0 x 1 E$ 0x2B 0xD9 0x88 0xA0 0xE9 0x7B 0xB3 0x6F 0x5D 0xB8 0x72 0xF1 0x04 0x95 0x6B 0xFB $0 x 15$ 0xE2 $0 x 83$ 0x63 0x19 0x06 0x30 0xB4 0xBA 0x3D $0 x 07$ 0x86 0xBF $0 x F 9$ 0x6D

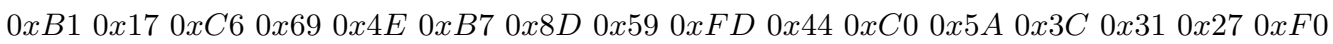
0x65 0xE1 0x62 0x3F 0xD2 0x4D 0xCF $0 x 78$ 0x7C $0 x 81$ 0x93 $0 x A 2 \quad 0 x B 0$ 0x0B $0 x F A$ 0x3B $0 x 940 x 250 x C 7$ 0x09 0x5C 0x22 0xF8 0x35 0x18 0x3E 0x97 0xEE 0x8A 0x52 0xE0 0x1A

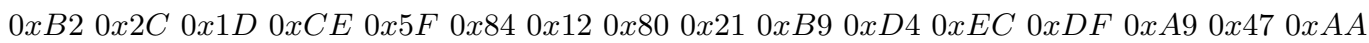

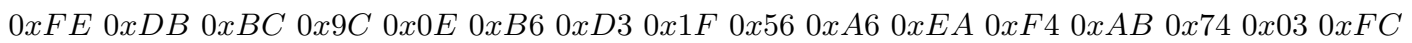
$0 x 010 x C 10 x 8 C$ 0x0D $0 x 85$ 0x7E $0 x 6 E$ 0x23 $0 x 67$ 0x8B $0 x C 9$ 0xAD $0 x 42$ 0xA7 $0 x D 10 x 9 E$ $0 x 750 x 46$ 0xDE $0 x 77$ 0xAF $0 x A E$ 0xC8 0x51 0x11 0x92 0x2E $0 x E 8$ 0x43 0x57 0xD6 $0 x E 6$

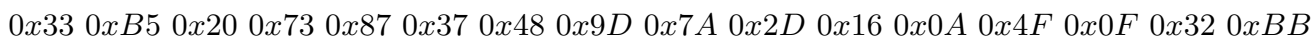
$0 x 660 x 530 x 290 x D 80 x C 2 \quad 0 x E 5 \quad 0 x 58$ 0x0C $0 x E 3 \quad 0 x 39$ 0x7D $0 x 130 x 360 x B E$ 0x7F $0 x 5 E$ 0xDC 0xC5 0x90 0xF2 0x55 0xD0 0x49 0xBD 0xA3 0x10 0x98 0xF3 0xEF 0x60 0x6A $0 x 24$

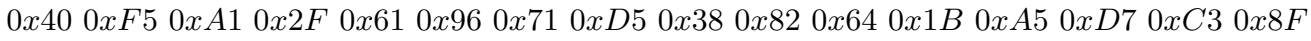
$0 x 140 x 3 A$ 0x6C $0 x 34$ 0x4A 0xDA $0 x 450 x 02 \quad 0 x 990 x 910 x 050 x 260 x C D \quad 0 x C B$ 0x41 $0 x E B$

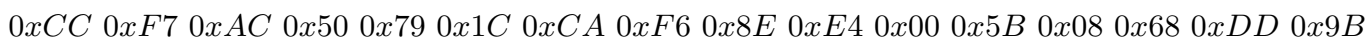

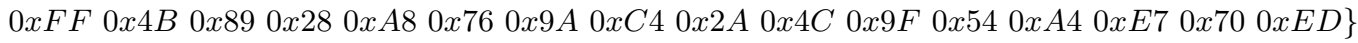

